NOUVELLE

\title{
Le réservoir viral dans l'infection par le VIH-2, modèle d'une infection rétrovirale atténuée
}

Assia Samri ${ }^{*}$, Charlotte Charpentier ${ }^{2 *}$, Rémi Cheynier ${ }^{3}$, Sophie Matheron ${ }^{4}$, Françoise Brun-Vézinet ${ }^{5 * \star}$, Brigitte Autran ${ }^{6 * \star}$ et le groupe d'étude ANRS CO5 IMMUNOVIR-2 ${ }^{\diamond}$

> Le virus de l'immunodéficience humaine de type 2 (VIH-2) [1] est un lentivirus responsable d'une infection moins grave que celle induite par le VIH de type 1 (VIH-1). L'infection par le VIH-2 est caractérisée par un maintien prolongé du nombre de lymphocytes $T$ $\mathrm{CD}^{+}$et par une charge virale plasmatique indétectable [2]. La progression plus lente de la maladie induite par le VIH-2 pourrait indiquer un équilibre hôte-pathogène plus favorable. La faible charge virale plasmatique, une des principales caractéristiques de I'infection par le VIH-2, a pour conséquence un taux de transmission réduit, et une concentration de l'infection en Afrique occidentale, où 1 à 2 millions de personnes pourraient être infectées [3]. Le génome du VIH-2 est proche de celui du VIH-1 (50\% d'identité de séquence nucléotidique) [4], et est presque identique à celui du virus de l'immunodéficience simien (VIS) des singes sooty mangabeys (sm) [5]. De plus, le $\mathrm{VIH}-2$, qui utilise les mêmes co-récepteurs CCR5 ( $C-C$ chemokine receptor 5$)$ et CXCR4 ( $c-x-c$ chemokine receptor 4$)$ que le $\mathrm{VIH}-1$ pour infecter les cellules [6], semble utiliser un plus large spectre de co-récepteurs alternatifs (CCRl à CCR8, CXCR6, GPRI5 [G

- Le groupe d'étude ANRS C05 IMMUNOVIR-2 :

Victor Appay, Brigitte Autran, Françoise Brun-Vézinet, Nathalie Chaghil, Charlotte Charpentier, Rémi Cheynier, Diane Descamps, Anne Hosmalin, Gianfranco Pancino, Nicolas Manel, Lucie Marchand, Sophie Matheron, Fideline Collin, Livia Pedroza-Martins, Asier Sàez-Cirion, Assia Samri, Rodolphe Thiebaut, et Vincent Vieillard. protein-coupled receptor 15], GPR1, APJ [putative receptor protein related to ATI ], CX3CRI (V28), CXCR5 et RDCl/ CXCR7) in vitro [7]. CXCR6 pourrait être un des co-récepteurs alternatifs majeur du VIH-2 car il serait préférentiellement utilisé chez les patients avirémiques infectés par le VIH-2 [8] ainsi que dans les infections par les lentivirus simiens non pathogènes [9]. Comme pour le VIH-1, l'infection par le VIH-2 semble aboutir à la constitution d'un réservoir de cellules infectées. Cependant, les mécanismes physiopathologiques expliquant les faibles charges virales du VIH-2 restent peu explorés. Le réservoir latent et inductible du VIH-1, intégré dans le génome cellulaire, est principalement localisé dans les lymphocytes $T \mathrm{CD}^{+}$à mémoire centrale ( $T$ central memory, TCM) au repos [10] et dans quelques monocytes et macrophages [11, 12]. Inversement, dans l'infection atténuée par VIS $S_{s m}$, la contribution des TCM au réservoir viral est plus faible que celle des cellules plus différenciées, du fait de leur faible expression du co-récepteur CCR5 [13]. De même, une infection plus réduite des TCM a été observée dans l'infection par le VIH-l des sujets «non progresseurs à long terme» (long-term non progressors, LTNP), porteurs des allèles protecteurs HLA (human leukocyte antigen) $B \star 27$ ou $B \star 57$ [14], ou chez les «contrôleurs » du VIH-l posttraitement [12].
${ }^{1}$ Sorbonne Université, Inserm U1135, Centre d'immunologie et des maladies infectieuses, Cimi-Paris, F-75013 Paris, France.

${ }^{2}$ Inserm, IAME, UMR 1137, Universités Paris Diderot et Paris Nord, Sorbonne Paris Cité ; Laboratoire de virologie, Hôpital Bichat, AP-HP, Paris, France.

${ }^{3}$ Université de Paris, Institut Cochin, Inserm U1016, CNRS UMR8104, F-75014 Paris, France. ${ }^{4}$ Inserm, IAME, UMR 1137, Universités Paris Diderot et Paris Nord, Sorbonne Paris Cité, Service des maladies infectieuses et tropicales, Hôpital Bichat, AP-HP, Paris, France.

${ }^{5}$ Université Paris7 Denis Diderot, Paris, France.

${ }^{6}$ Sorbonne Université, Inserm U1135, Centre d'immunologie et des maladies infectieuses, Cimi-Paris, AP-HP, Hôpital universitaire Pitié-Salpêtrière, F-75013 Paris, France.

* Ces auteures ont contribué de façon égale à ce travail.

** Ces auteures ont contribué de façon égale à ce travail.

assia.samri@upmc.fr

Afin de mieux définir les caractéristiques du réservoir du VIH-2, nous avons analysé sa distribution parmi les cellules sanguines périphériques $\mathrm{CD}^{+}$provenant d'individus suivis dans la cohorte française ANRS-VIH-2 CO5 [15]. Nous avons cherché à savoir si les monocytes étaient infectés in vivo, et avons testé l'hypothèse d'une infection plus restreinte des TCM.

Distribution préférentielle du réservoir du VIH-2 dans les lymphocytes $\mathrm{T} \mathrm{CD4} 4^{+}$à mémoire transitionnelle ( $T$ transitional memory, $\mathrm{TM}$ ) et absence d'infection des monocytes

Des sujets infectés par le VIH-2 et n'ayant reçu aucun traitement antirétroviral (12 individus «non progresseurs», et 2 《progresseurs») ont été inclus dans une étude prospective [15]. Les monocytes ainsi que des sous-populations de lymphocytes $T$ $\mathrm{CD}^{+}$au repos - naïfs TN ( $\mathrm{T}$ naive), à mémoire centrale TCM, à mémoire transitionnelle TTM, et à mémoire effectrice TEM ( $T$ effector memory) 


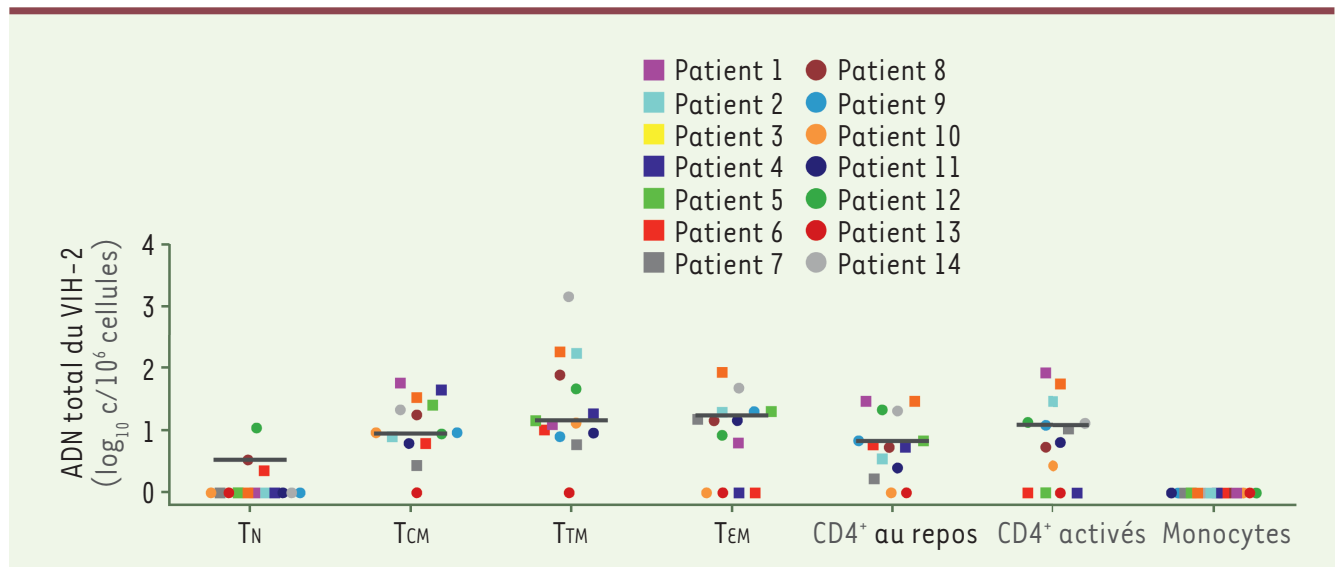

Figure 1. Distribution de I'ADN total du VIH-2 dans les monocytes et différentes sous-populations de lymphocytes $T$. L'ADN total du VIH-2 a été quantifié dans différentes populations cellulaires triées à partir des cellules mononucléées du sang périphérique (CMNS) chez 14 sujets infectés par le VIH-2. Sept populations ou sous-populations cel-

lulaires ont été analysées : les populations de monocytes ( $\left.C D 3^{-} \mathrm{CD} 4^{-} \mathrm{CD} 14^{+}\right)$, de lymphocytes $\mathrm{TCD}^{+}$activés $\left(\mathrm{CD} 25^{+} \mathrm{CD} 69^{+} \mathrm{HLADR} \mathrm{R}^{+}\right)$, de lymphocytes T CD4 $4^{+}$au repos (CD25- $\mathrm{CD} 69^{-} \mathrm{HLADR}^{-}$), les sous-populations de lymphocytes T CD4 $4^{+}$au repos : les lymphocytes T naïfs $\left(\mathrm{TN} ; \mathrm{CD} 45 \mathrm{RA}{ }^{+} \mathrm{CCR} 7^{+} \mathrm{CD} 27^{+}\right)$, les lymphocyte Tà mémoire centrale (TCM ; CD45RA- CCR7 $7^{+}$CD27 ${ }^{+}$), les lymphocytes T à mémoire transitionnelle (TTM ; CD45RA ${ }^{-}$CCR7- $\left.C D 27^{+}\right)$et les lymphocytes T à mémoire effectrice (TEM ; CD45RA- CCR7- CD27-). Les résultats sont exprimés en log 10 du nombre de copies (c) d'ADN du VIH-2 par million de cellules et les valeurs médianes sont indiquées par des barres horizontales. Chaque symbole représente un sujet.

- ont été triés à partir des cellules mononucléées du sang (CMNS). L'ADN du VIH-2 a été quantifié par PCR (polymerase chain reaction) en temps réel dans chaque sous-population triée. Nous avons ainsi montré que le réservoir du VIH-2 diffère du réservoir du VIH-l par une absence totale d'infection des monocytes [11, 12, 14] et une infection prédominante des TTM plutôt que des TCM (Figure 1), confirmant donc l'hypothèse d'une infection réduite des TCM, comme cela est observé dans le modèle d'infection non pathogène de singes sooty mangabeys par le VIS $_{\mathrm{sm}}$ [13], ainsi que chez les sujets LTNP [14] ou les contrôleurs post-traitement infectés parle VIH-1 [12].

Faible inductibilité du VIH-2 dans les cellules CD4 ${ }^{+}$circulantes

Dans notre étude, nous n'avons pu détecter que de faibles niveaux d'ADN du VIH-2 chez les 14 sujets infectés par le VIH-2 testés, cette infection n'étant quantifiable que chez 5 d'entre eux. De plus, nous n'avons pu réactiver in vitro la production virale dans les cellules mononucléées du sang que chez 3 individus, confirmant la faible productivité, déjà connue, du
VIH-2. Bien que ces résultats puissent refléter les caractéristiques de ces sujets majoritairement «non progresseurs», des résultats identiques ont été observés chez les 2 sujets «progresseurs » bien qu'ayant une faible charge virale VIH-2 plasmatique.

La distribution du réservoir du VIH-2 dans les sous-populations de lymphocytes T CD4 ${ }^{+}$mémoires est associée à un déséquilibre de l'expression des gènes CXCR6 et TRIM5 $\alpha$

Afin de mieux comprendre les déterminants de la distribution atypique du réservoir du VIH-2, nous avons analysé le profil transcriptomique des souspopulations $\mathrm{T} \mathrm{CD}^{+}$triées, concernant un total de 96 gènes associés à I'inflammation, aux cytokines, aux chimiokines, et aux voies de signalisation de l'interféron, avec un intérêt particulier pour les co-récepteurs alternatifs du VIH tels que CXCR6 [8] et pour des facteurs de restriction potentiels du VIH-2 tels que TRIM $5 \alpha$ (tripartite motif protein $5 \alpha$ ). L'ADN du VIH-2 n'étant détectable que dans les cellules TTM et TCM, nous avons concentré notre analyse sur ces deux sous-populations. Les résultats obte- nus ont permis de montrer que le gène CXCR6 était moins exprimé dans les TCM que dans les TTM (Figure 2), et nous avons pu confirmer cette différence en analysant l'expression de la protéine CXCR6 par cytométrie en flux (Figure 3A). Comme il a été récemment montré que l'utilisation du CXCR6 caractérise l'infection par les lentivirus non pathogènes [9] et qu'elle est abrogée par la présence d'une proline en position 326 de la boucle V3 de la glycoprotéine d'enveloppe gp 105, nous avons examiné les 259 séquences de gpl05 du VIH-2 dont nous disposions pour la cohorte ANRS-VIH-2, mais aucune de ces séquences ne comportait une proline à cette position.

L'analyse transcriptomique a également montré que le gène TRIM5 $\alpha$ était plus exprimé dans les TCM que dans les TTM (Figure 2), mais l'analyse de l'expression de la protéine par cytométrie en flux n'a pas permis de confirmer cette différence (Figure $3 B$ ). Alors que TRIM5 $\alpha$ n'a pas d'effet sur l'infection par le VIH-1, c'est un puissant facteur de restriction du VIS. Du fait de la similitude des séquences de capside entre $\mathrm{VIS}_{\mathrm{sm}}$ et $\mathrm{VIH}-2$, le VIH-2 montre des niveaux élevés de sensibilité au TRIM5 $\alpha$ humain [16]. TRIM5 $\alpha$ pourrait 


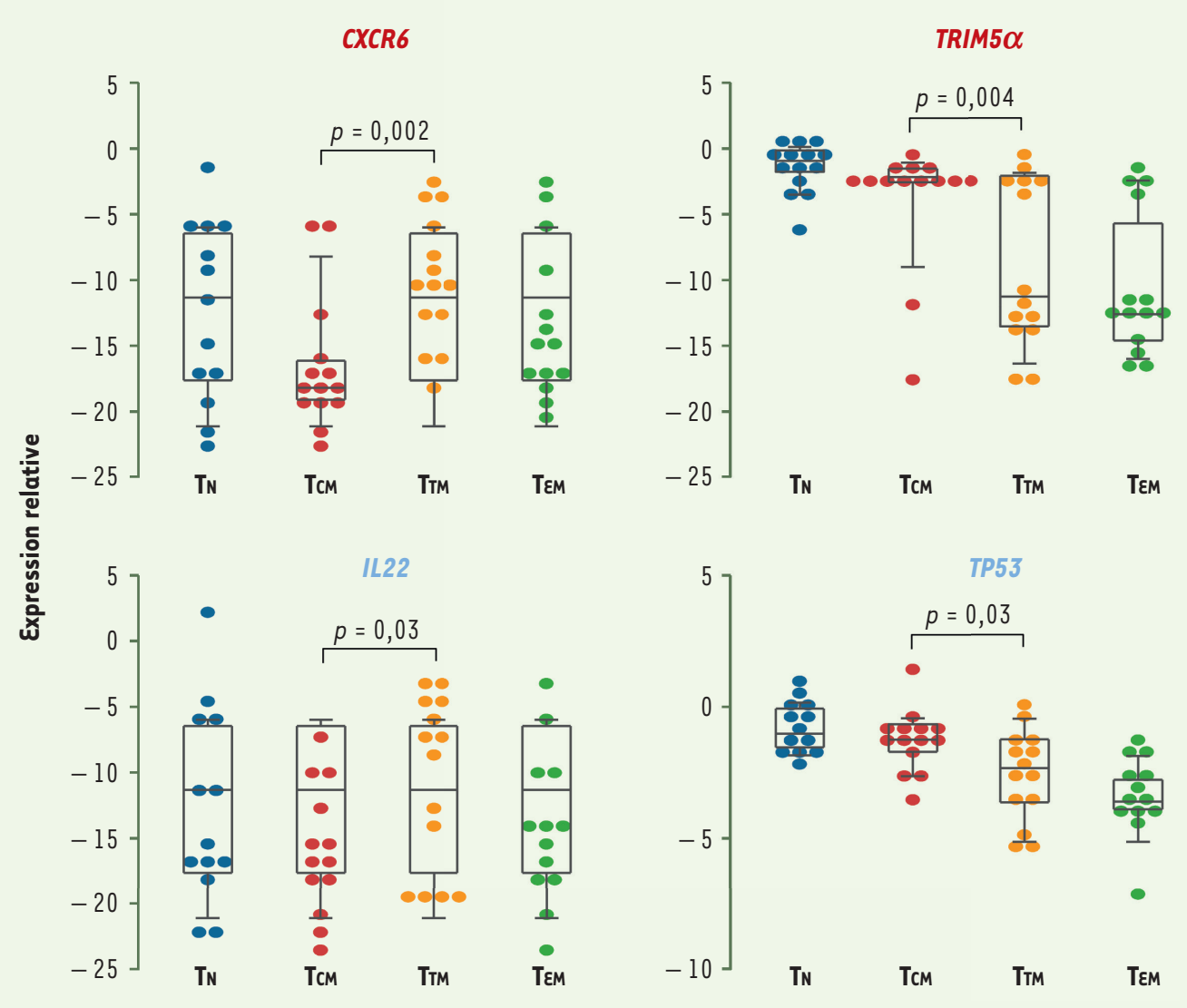

Figure 2. Analyse transcriptomique des monocytes et des sous-populations de lymphocytes $T$ CD4 $4^{*}$ au repos. Une analyse des transcrits de 96 gènes a été réalisée dans les monocytes $\left(C D 3^{-} C D 4^{-} C D 14^{+}\right)$et les sous-populations de lymphocytes T CD4+ au repos : les lymphocytes T naïfs (TN ; CD45RA ${ }^{+} C C R 7^{+} C D 27^{+}$), les lymphocytes $T$ à mémoire centrale (TCM ; CD45RA- CCR7 $7^{+} C D 27^{+}$), les lymphocytes $T$ à mémoire transitionnelle (TTM ; CD45RA- $C C R 7^{-}$CD27 $7^{+}$), et les lymphocytes T à mémoire effectrice (TEM ; CD45RA CCR7- CD27-) de 14 sujets infectés par le VIH-2. La figure montre l'expression relative des gènes CXCR6, TRIM5 $\alpha$, IL22, et TP53 dans les sous-populations de lymphocytes T CD $4^{+}$au repos. L'expression de ces gènes a été normalisée par rapport à celle du gène «de ménage » RPS14.

donc également contribuer à réduire l'infection par le VIH-2 des TCM par rapport aux TTM.

Ainsi, nos résultats indiquent que la faible expression de CXCR6 et, dans une moindre mesure, la surexpression de TRIM $5 \alpha$ par les TCM [15], pourraient expliquer les différences de contribution des différentes sous-populations de cellules $\mathrm{T} C D 4^{+}$mémoires aux réservoirs latents du VIH-2 et du VIH-1.

En conclusion, ce travail a identifié deux éléments caractéristiques de la distribution du réservoir du VIH-2 dans les cellules mononucléées du sang périphérique de personnes infectées par ce virus, progressant ou non dans la maladie. L'absence d'infection des monocytes par le VIH-2, alors que ces cellules servent fréquemment de réservoir au VIH-1, suggère que le virus VIH-2, dérivé du VIS, est mal adapté aux cellules de la lignée myéloïde. Par ailleurs, la plus faible infection des lymphocytes $\mathrm{T} \mathrm{CD4} 4^{+}$à mémoire centrale, conséquence d'une moindre expression du co-récepteur CXCR6, et peut-être de la présence plus importante du facteur de restriction TRIM $5 \alpha$, suggère que le VIH-2 pourrait également être moins adapté aux lymphocytes $T \mathrm{CD} 4^{+}$ à mémoire centrale que ne l'est le VIH-1. L'ensemble de ces résultats suggère que cette distribution particulière du réservoir du VIH-2 pourrait être liée en partie à des facteurs clés de l'hôte, jetant ainsi un nouvel éclairage sur la moindre pathogénicité du VIH-2, encore mal comprise. Ce travail s'inscrit dans le cadre du programme ANRS Immunovir2, qui a également permis de montrer que des réponses immunes puissantes, par l'intermédiaire des cellules NK (natural killer) ou des lymphocytes T CD $8^{+}$, pouvaient contribuer au contrôle prolongé de l'infection par le VIH-2, suggérant que plusieurs modalités de la relation hôte-virus régissent la faible pathogénicité du VIH-2. $\diamond$

Viral reservoir in HIV-2 infection: a model for attenuated retroviral infection 

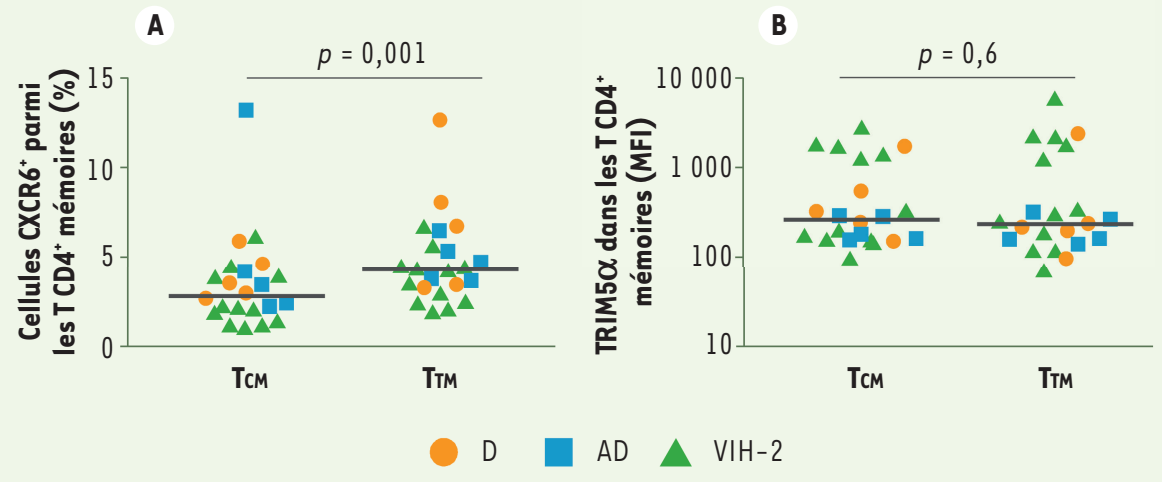

Figure 3. Analyse de l'expression des protéines CXCR6 et TRIM5 $\alpha$ dans les lymphocytes T CD4 ${ }^{+}$TCM et TTM par cytométrie en flux. Analyse des lymphocytes $T$ $\mathrm{CD4}^{+}$à mémoire centrale (TCM ; CD45RA- CCR7 ${ }^{+}$ $\left.\mathrm{CD} 27^{+}\right)$et transitionnels (TTM ; CD45RA- CCR7 ${ }^{-}$ $\mathrm{CD} 27^{+}$) par cytométrie en flux chez 12 sujets infectés par le VIH-2 (triangles),

5 donneurs ouest-africains non-infectés ( $A D$, carrés) et 4 autres donneurs non-infectés de l'Établissement français du sang ( $D$, ronds). La figure montre le pourcentage de cellules CXCR6 ${ }^{+}$parmi les lymphocytes T CD4 ${ }^{+}$mémoire en A, et l'intensité moyenne de fluorescence (MFI) de TRIM5 $\alpha$ intracellulaire en $B$. Chaque symbole représente un sujet, et les valeurs médianes pour chaque groupe sont indiquées par des barres horizontales.

\section{LIENS D'INTÉRÊT}

Les auteurs déclarent n'avoir aucun lien d'intérêt concernant les données publiées dans cet article.

\section{RÉFÉRENCES}

1. Clavel F, Guetard D, Brun-Vezinet F, et al. Isolation of a new human retrovirus from West African patients with AIDS. Science $1986 ; 233: 343-6$.

2. Nyamweya $S$, Hegedus $A$, Jaye $A$, et al. Comparing HIV-1 and HIV-2 infection: lessons for viral immunopathogenesis. Rev Med Virol 2013 ; 23 : 221 40.

3. Visseaux B, Damond F, Matheron S, et al. HIV-2 molecular epidemiology. Infect Genet Evol 2016 ; 46 : 233-40.

4. De Silva TI, Cotten M, Rowland-Jones SL. HIV-2: the forgotten AIDS virus. Trends Microbiol 2008 ; 16 : 588 95.

5. Santiago ML, Range F, Keele BF, et al. Simian immunodeficiency virus infection in free-ranging sooty mangabeys (Cercocebus atys atys) from the Tai Forest, Côte d'Ivoire: implications for the origin of epidemic human immunodeficiency virus type 2.J Virol $2005 ; 79$ : 12515-27.

6. Sol N, Ferchal F, Braun J, et al. Usage of the coreceptors CCR -5, CCR-3, and CXCR- 4 by primary and cell line-adapted human immunodeficiency virus type 2. Jirol $1997 ; 71: 8237-44$.

7. Shimizu N, Soda Y, Kanbe K, et al. A putative $G$ protein-coupled receptor, $\mathrm{RDCl}$, is a novel coreceptor for human and simian immunodeficiency viruses. J Virol $2000 ; 74: 619-26$.

8. Blaak H, Boers PH, Gruters RA, et al. CCR5, GPRI5, and CXCR6 are major coreceptors of human immunodeficiency virus type 2 variants isolated from individuals with and without plasma viremia. J Virol $2005 ; 79: 1686-700$

9. Wetzel KS, Yi Y, Yadav A, et al. Loss of CXCR6 coreceptor usage characterizes pathogenic lentiviruses. PLoS Pathog 2018 ; 14 : e1007003.

10. Chomont N, El-Far M, Ancuta P, et al. HIV reservoir size and persistence are driven by $T$ cell survival and homeostatic proliferation. Nat Med 2009 ; 15 : 893900.

11. Bacchus C, Cheret A, Avettand-Fenoel V, et al. A single HIV-l cluster and a skewed immune homeostasis drive the early spread of HIV among resting $\mathrm{CD}^{+}$cel subsets within one month post-infection. PLoS One 2013 ; 8 : e64219.

12. Saez-Cirion A, Bacchus C, Hocqueloux L, et al. Post-treatment HIV-1 controllers with a long-term virological remission after the interruption of early initiated antiretroviral therapy ANRS VISCONTI Study. PLoS Pathog 2013 ; 9 : el003211.

13. Paiardini M, Cervasi B, Reyes-Aviles $\varepsilon$, et al. Low levels of SIV infection in sooty mangabey central memory $C D 4^{+} T$ cells are associated with limited CCR5 expression. Nat Med 2011; $17: 830-6$.

14. Descours B, Avettand-Fenoel V, Blanc C, et al. Immune responses driven by protective human leukocyte antigen alleles from long-term nonprogressors are associated with low HIV reservoir in central memory $\mathrm{CD}^{+} \mathrm{T}$ cells. Clin Infect Dis $2012 ; 54$ : 1495-503.

15. Samri A, Charpentier C, Diallo MS, et al. Limited HIV-2 reservoirs in central-memory $\mathrm{CD}^{+} \mathrm{T}$-cells associated to CXCR6 co-receptor expression in attenuated HIV-2 infection. PLoS Pathog 2019 ; 15 : el007758.

16. Takeuchi JS, Perche B, Migraine J, et al. High level of susceptibility to human TRIM $5 \alpha$ conferred by HIV-2 capsid sequences. Retrovirology $2013 ; 10: 50$.

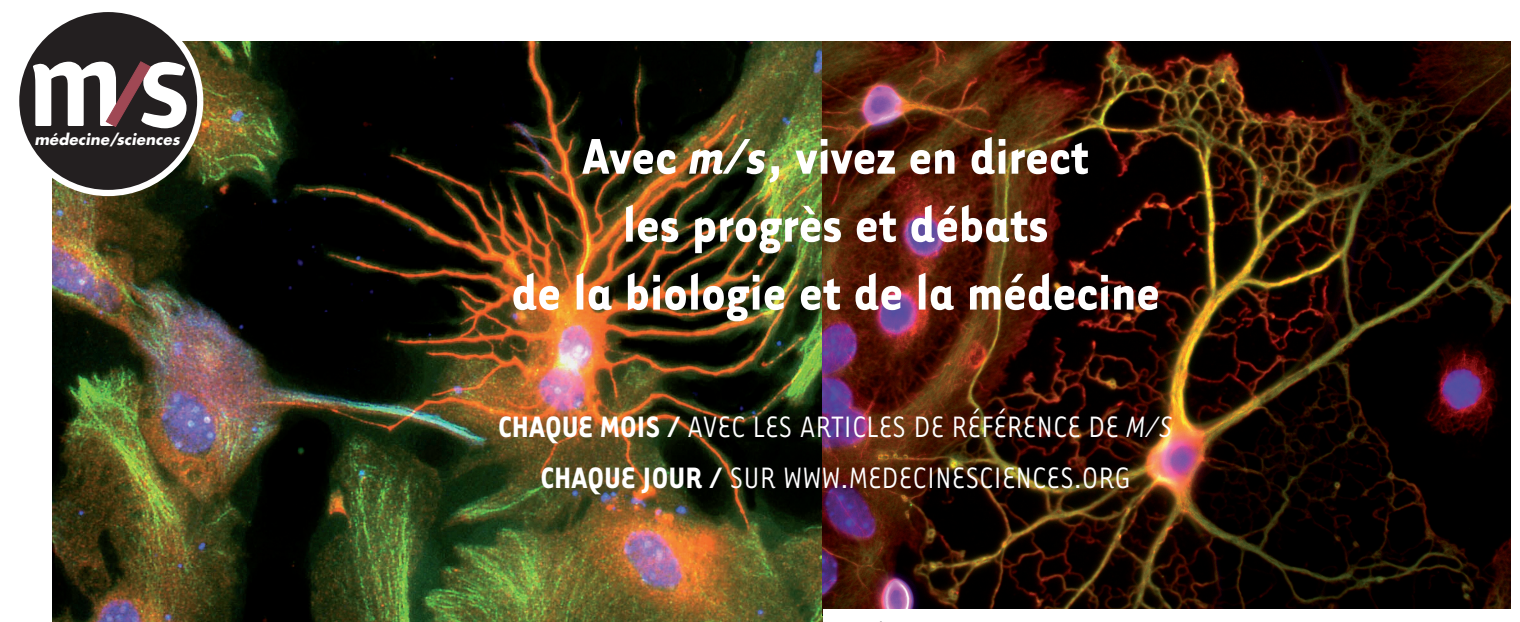

Abonnez-vous sur

www.medecinesciences.org 